\title{
Designing Physics Game to Support Inquiry Learning and to Promote Retrieval Practice
}

\author{
Danu Pranantha ${ }^{1,2}$, Wei Chen ${ }^{1}$, Francesco Bellotti ${ }^{2}$, Erik Van Der Spek ${ }^{1}$, Alessandro DeGloria ${ }^{2}$ \\ and Matthias Rauterberg ${ }^{1}$ \\ ${ }^{1}$ Industrial Design Department, Eindhoven University of Technology, Eindhoven, The Netherlands \\ ${ }^{2}$ DITEN, University of Genoa, Genoa, Italy \\ \{d.pranantha,w.chen\}@tue.nl,franz@elios.unige.it,e.d.v.d.spek@tue.nl,adg@elios.unige.it,g.w.m.rauterberg@tue.nl
}

Keywords: $\quad$ Physics Games, Game Design, Simulation, Pseudo-tutor, Easy Authoring.

\begin{abstract}
Instruction in physics aims at achieving two goals: the acquisition of body of knowledge and problem solving skills in physics. This requires students to connect physical phenomena, physics principles, and physics symbols. Computer simulation provides students with graphical model that unites phenomenon and principles in physics. However, such minimally guided approach may harm learning since it overburdens the working memory. Also, simulation is inadequate in promoting problem solving skills since students need to exercise with a variety of physics problems. Intelligent tutoring systems (ITS), in contrast, train students in solving physics problems. In this paper, we designed an online puzzle game in physics that combines simulation and pseudotutor (namely QTut). We addressed three challenges: extensibility, scalability, and reusability in designing our game. We conducted usability tests with 10 participants on the game prototype to study the user performances and perceptions for improvement. The results indicate the game as educative and moderately entertaining. The use of scaffolding in the game positively contributed to the game learning experience. Moreover, the game GUI expressed information well that made the game understandable, even with little instructions.
\end{abstract}

\section{INTRODUCTION}

Instruction in physics aims at achieving two goals: the acquisition of body of knowledge and the ability to solve quantitative problems in physics. To achieve the learning goals, physics instructions should examine the knowledge structure of physics. Physics organize the body of knowledge into three levels: the macroscopic level corresponds to physical objects, their properties and behaviour; the microscopic level explains the macroscopic level using concepts, theories and principles of physics; and the symbolic level represents the concepts of physics as mathematical formulae (Johnstone, 1991). Consequently, physics instructions need to advocate the connection of those levels to the students.

Lack of knowledge and/or misconceptions at the microscopic level leads students to difficulties in solving physics problems (Heyworth, 1999). The use of concrete models, analogies and graphics may help students to overcome those difficulties. In inquiry learning, computer simulation graphically models physical objects and unites the macroscopic, the microscopic, and the symbolic levels together. This approach urges students to actively seek questions, explore the simulation environment, and discover knowledge based on their observations.

However, such minimally guided approach may harm learning since it does not align with working memory limitations (Kirschner and Clark, 2006). This, to an extent, necessitates the use of scaffolding, which is essential particularly for inquiry learning (Jong, 2006). The use of scaffolding reduces $\operatorname{cog}$ nitive load of the students when using computer simulation. A meta analysis also supported the use of explicit instructions in learning (Alfieri et al., 2011). Moreover, guided inquiry learning also helps students to plan their simulation experiments (Bonestroo and de Jong, 2012).

Nevertheless, using simulation alone is inadequate in promoting problem solving skills. It is also important for students to exercise with a variety of physics problems and to perform retrieval practices at microscopic and symbolic levels (Karpicke and Blunt, 2011). Intelligent tutoring systems (ITS) train students in problem solving skills using apprenticeship and problem solving models (Woolf, 2009).

Given the advantages of simulation and tutoring 
system, we combined simulation and tutoring system into a serious game for learning physics. Serious games have potentials in instruction particularly for engaging the audience in challenging and contextualized activities; and students are encouraged to query information, apply knowledge and practice skills (Wouters et al., 2013; Connolly et al., 2012). We designed the game as an online physics game accompanied by a pseudo tutor to support learning. The game graphically simulates the macroscopic level whereas the example-tracing tutor (pseudo tutor) explains the physical phenomenon at both microscopic and symbolic levels. We conducted usability tests on the game prototype to collect user performance and perception of the game as a learning media.

The remainder of the paper is organized as follows. Section 2 relates our work with the current state of the arts, whereas Section 3 explains our game in details. Section 4 discusses about the usability tests, and Section 5 concludes our paper.

\section{RELATED WORKS}

Constructivist teaching has the greatest potential to enhance learning where learners actively construct knowledge through inquiry, apprenticeship, and collaboration (Woolf, 2009). In physics, constructivist teaching uses computer simulation to challenge learners constructing their own questions, exploring the simulation environment, and inferring knowledge similar to scientists.

Numerous computer simulations for learning physics are available in the market. For instance, PhET project provides a variety of interactive physics simulations (Perkins et al., 2006). The PhET project investigated several design factors of engaging and effective simulation (Adams et al., 2008a; Adams et al., 2008b). The finding suggests that providing driving questions encourage students to explore the simulation (Adams et al., 2008c). Furthermore, balanced challenges in form of affordance, constraints, analogies, and the use of multiple representations maintain students engagement (Podolefsky et al., 2010).

Several studies showed that the use of scaffolding improves the effectiveness of simulation. For instance, traditional instructions enhanced learners understanding of the simulation (Kolloffel and de Jong, 2013) and helped them to plan their experiments (Bonestroo and de Jong, 2012). The use of concept mapping with simulation enhanced deep learning (Gijlers and de Jong, 2013).

However, computer simulation overlooked problem solving in physics that most students find it dif- ficult. In contrast, Intelligent Tutoring Systems (ITS) and Fading Worked Example (FWE) can be used to nurture problem solving skills. For instance, Andes tutor trained students on solving physics problems (Vanlehn et al., 2005) improved the average exam score of the students. Likewise, FWE supports effective learning but combining ITS and FWE did not contribute to better learning (McLaren et al., 2008).

Combining a physics simulation with a tutoring system may provide students with a graphical tool for exploration (the macroscopic level) and a training tool for problem solving (the microscopic and the symbolic levels). A practicable approach is using serious games to combine both simulation and tutoring systems. Serious games have strengths to appeal and to motivate students (Connolly et al., 2012). Meta analysis showed that games can be more effective than traditional instructions, but only when considering working memory limitations (Wouters et al., 2013).

In this paper, we created an online puzzle game in physics that uses simulation to represent physical objects at the macroscopic level and a pseudo tutor (namely QTut) to explain physical phenomenon at the microscopic and the symbolic levels. QTut provides students with hints and feedback; and it responds to student queries. As a prototype, we created a physics game for bachelor degree students. We conducted usability tests to study the user performances and perceptions of the game for improvement.

\section{GAME SYSTEM DESIGN AND DEVELOPMENT}

\subsection{Design Considerations}

The game system was developed using bottom up approach by identifying challenges, devising solutions, designing each solution as a module, and integrating the modules into complete system. The graphical user interface (GUI) and the gameplay emphasize the use of scaffoldings in form of driving questions, informative feedbacks, user queries, and QTut responses.

The game was implemented using HTML5 and JavaScript for rich web experiences and JSON (JavaScript Object Notation) for lightweight data storage. We followed rapid prototyping to iteratively create prototypes over short period. 


\subsection{Identifying and Addressing Challenges}

We considered three challenges in developing the game system: extensibility refers to the ease to produce a variety of games for different topics, scalability means the ease to attach new modules to the system, and reusability corresponds to the use of some modules for other purposes.

\subsubsection{Extensibility}

\section{A. Game Level and Game Format}

We created game level and game format to allow extensibility (Pranantha et al., 2012). The game level clusters learning topics into levels based on their complexity. The game format sets each game level as series of tasks a puzzle set drawn from the database (a JSON file). A task or a task item is either a closed ended question about a simulated event or an action request in the simulation area.

Figure 1 described a puzzle set that consists of several task items. Each task item has two types of data: the scaffolding data and the simulation data. The scaffolding data (Program 1) has an id, a question, a list of feedbacks, a sequence of possible answers, and an index of correct answer. Subsequently, the simulation data (Program 2) includes a collection of objects and a list of available responses to the action request.

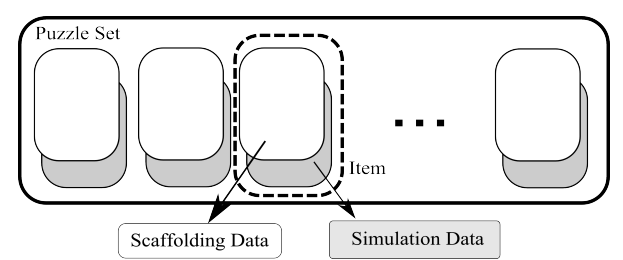

Figure 1: A puzzle set and a task item.

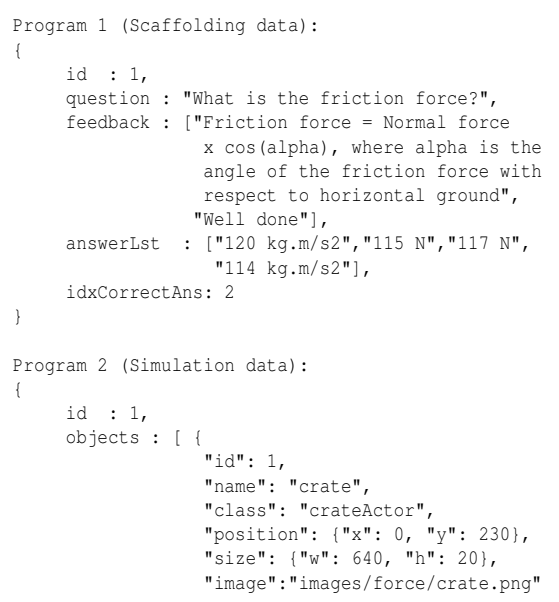

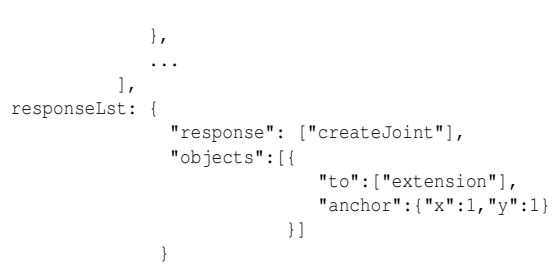

Using the game format, a game consists of a sequence of inter-related tasks can be easily created to learn problem solving skills. Some tasks can be recalled several times to promote retrieval practice which is essential for learning (Karpicke and Blunt, 2011).

\section{B. Pseudo Tutor}

Beside the scaffolding data in the task item, we created QTut, a pseudo tutor. QTut allows students to query some information in relation to the task at hand.

To support the extensibility of QTut, we created knowledge triplet (Qs, R, DA), where Qs refers to a list of query samples; $\mathrm{R}$ represents a response to a list of query samples Qs; and DA denotes dialog act (Program 3). The knowledge triplet (subsequently called triplet) represents QTUt knowledge on learning topics. Consequently, the number of triplets is contingent to the coverage of the learning topics in the game.

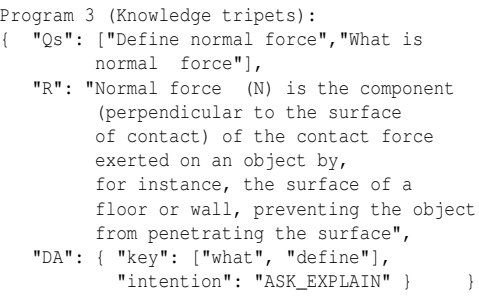

We use the triplets to construct a $\mathrm{N}$-gram term frequency - inverse document frequency (TF-IDF) table (Table 1) that measures how concentrated the occurrence of a given word in a collection of triplets. Words with high TF-IDF numbers imply a strong relationship with the triplet they appear in, suggesting that if that word were to appear in a query, the triplet could be of interest to the student.

Table 1: An example of N-gram TF-IDF table with 2 triplets.

\begin{tabular}{|l|r|r|}
\hline N-gram words & $\begin{array}{c}\text { TF-IDF of } \\
\text { triplet 1 }\end{array}$ & $\begin{array}{c}\text { TF-IDF of } \\
\text { triplet 2 }\end{array}$ \\
\hline Net force & 0.40 & 0 \\
\hline Normal force & 0 & 0.4 \\
\hline Force & 0.10 & 0.10 \\
\hline
\end{tabular}

TF-IDF is computed as follows. Suppose we have a collection of $N$ triplets. Define $f_{i j}$ to be the frequency (number of occurrences) of term $i$ in triplet $j$. Then, define $T F_{i j}$ to be $f_{i j}$ normalized by dividing 
it with the maximum number of occurrences of any term in the same triplet (1) (Rajaraman and Ullman, 2011).

$$
T F_{i j}=\frac{f_{i j}}{\max _{k}\left(f_{k j}\right)}
$$

Whereas the IDF for a term is dened as follows. Suppose term $i$ appears in $n_{i}$ of the $N$ triplets in the collection. Then,

$$
I D F_{i}=\log _{2} \frac{N}{n_{i}}
$$

The TF-IDF score for term $i$ in triplet $j$ is then computed as

$$
T F-I D F_{i j}=T F_{i j} . I D F i
$$

We developed a Naive Bayes classifier to determine the similarity between a query and the triplets using TF-IDF information (Manning et al., 2008). QTut subsequently ranks the similarity values in descending order and removes triplets that have similarity values below a certain threshold. QTut performs intention matching on the DA of the remaining triplets with the following rules: if it finds a match, then returns the corresponding triplet; otherwise, returns the triplet with the highest similarity value.

\subsubsection{Scalability and Reusability}

To facilitate scalability and reusability, the game system is divided into functionality modules (Figure 2): a) tutoring module delivers questions, provides hints and feedbacks, and responds to queries; $b$ ) physics simulation module handles all graphical events based on laws in physics; $c$ ) delivery module draws a task item from the puzzle set either in random, sequential, or difficulty based order; and $d$ ) data module accesses, organizes, and manipulates game database (i.e., game contents, game configuration, and user log).

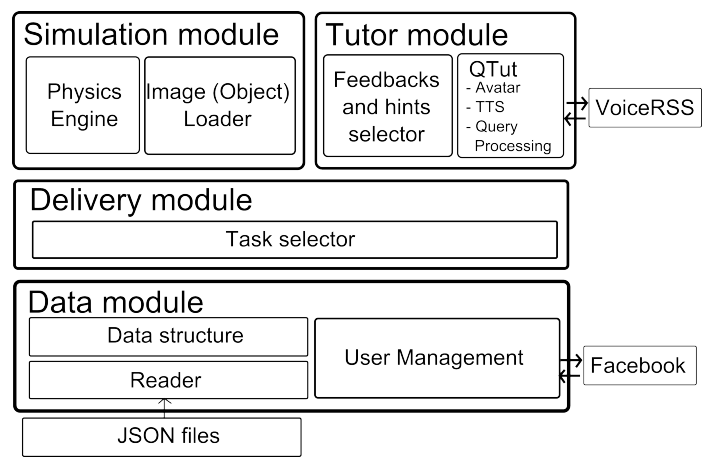

Figure 2: A stack of modules as a complete system architecture.
QTut (in the tutoring module) has two modes: text only and text with speech. We use a free textto-speech (TTS) web service ${ }^{1}$ to convert texts into speeches for the latter mode (Figure 3). QTut sends the texts to the TTS web API using HTTP GET and the TTS web API subsequently synthesizes the speeches and sends them to QTut.

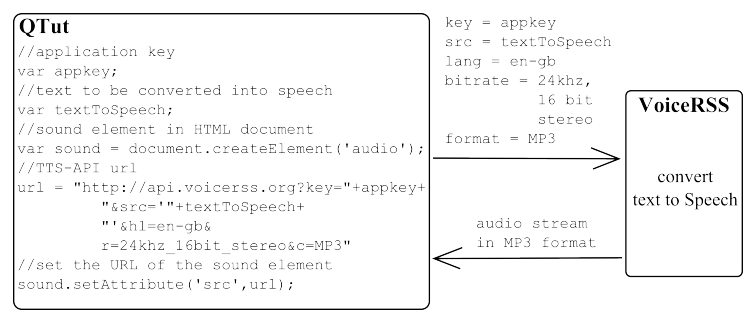

Figure 3: Converting Text to Speech.

To minimize the needs of user management and to support the game distribution, the system is connected to a social networking platform (Facebook) using Facebook Javascript API ${ }^{2}$. The system extracts user information on Facebook to be stored into the database.

\subsection{Gameplay}

Gameplay refers to formalized interaction that occurs when players follow the rules of a game and experience its system through play (Salen and Zimmerman, 2003). Game rules are, therefore, the heart of any game which distinguish game (e.g., a football match) from play (e.g., two kids pass a ball to each other). Furthermore, instruction process -presenting new information, integrating new knowledge, and connecting new knowledge with prior knowledge- is essential in learning (Ferguson-hessler and de Jong, 1991). In serious games, game rules and instruction process are entwined to deliver fun yet educative gameplay. The rules should be easy to comprehend and the instruction process should consider working memory limitations.

The game rules are as follows. All game levels are initially locked except at the base level (level 1). For simplicity, all task items in a level have the same weight for scoring. However, each level has three most difficult task items which each is indicated by a star. If a student answers a starred task item, he will receive one star.

A level has a topic related to its preceding and succeeding levels. For instance, force and torque can be

\footnotetext{
${ }^{1}$ VoiceRSS Text To Speech (http://voicerss.org/)

${ }^{2}$ Facebook Developer API (https://developers. facebook.com/)
} 
two successive levels. If a level is unrelated to its preceding, the QTut presents an introduction to denote topic transition. A student may progress to a level (i.e., unlock a level) if he has passed its preceding level. A student completes a level if he earns at least two stars and scores above a certain threshold. During the game, a student may query QTut about concepts, formulas, and terminologies. The game rules do not impose timeout but we use the timer for logging purpose.

\subsection{Graphical User Interface (GUI)}

The layout of the game GUI was designed using grid systems to group all elements according to their functionalities. This allows the game users to easily comprehend the interface (Elam, 2004). Figure 4 (left) shows the wireframe of the game GUI: tutor area on the top right consists of a tutor avatar and an input text to enter query for the tutor, information area on the middle presents feedback and task from the tutor, and simulation area on the bottom plays physics events. The final GUI of the Physics game prototype is shown in Figure 4 (right).

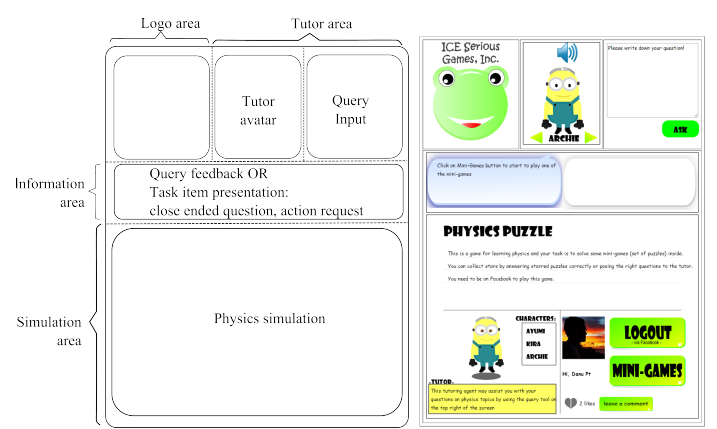

Figure 4: the wireframe of the game GUI (left) and the game GUI (right)

The GUI elements (e.g., buttons and playable objects) use the feedforward and feedback concept to allow intuitive interaction. Feedforward is the information that occurs during or after user action, for instance, on-screen messages indicating what to do. Feedback is 'the return of information about the result of a process or activity' (Wensveen et al., 2004). For instance, clicking on a button opens a new window.

Figure 5 shows the use of feedforward and feedback in a Logout button. Feedforward conveys an implicit message that the logout button is click-able by changing its color upon mouse-over event; and the feedback responds to user action (a click) by changing the logout button into a login button. Feedforward is also used to help students in problem solving. For instance, a calculator button appears if a task item

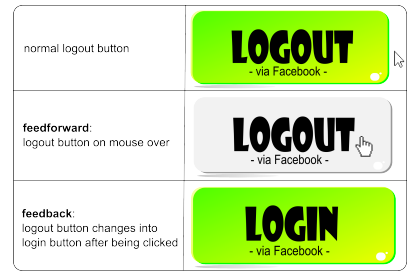

Figure 5: An example of feedforward and feedback in a Logout button.

asks student to calculate force. The physics simulation shows a ruler if student needs to measure length or distance.

\subsection{The Physics Game Prototype}

The Physics game prototype, intended for bachelor degree students, has two levels: force and torque. The first level consists of nine close ended questions. The questions are either conceptual or quantitative problems. The second level has six action requests that demands student to interact with objects in the simulation area. Figure 6 (top-left) shows a list of game levels where all levels are locked except level 1 (force). Figure 6 (top-right) shows a task item in the first level that asks about stationary state. Figure 6 (bottom) shows a task item in the second level that demands student to balance the mobile toy. Each correct answer is awarded with ten points and a starif the task item is a starred task item. A student passes a level if he earns two stars (three stars are available in each level) and scores above $50 \%$ (i.e., 50 points for level 1 and 30 points for level 2).

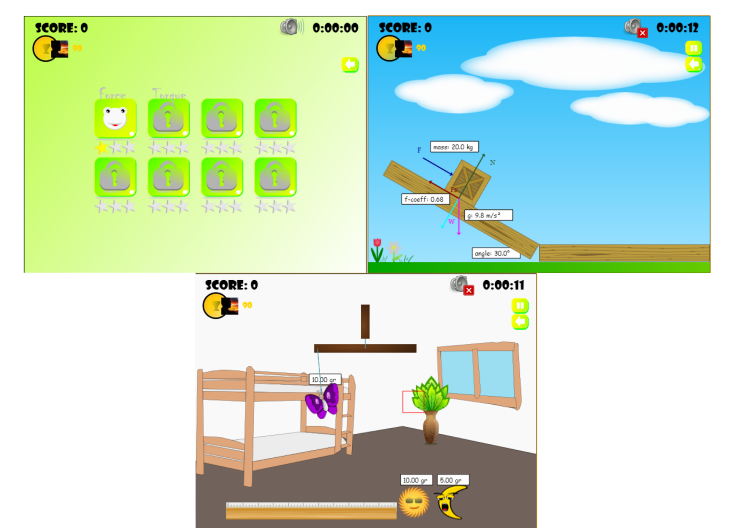

Figure 6: locked level (top-left), level 1 (top-right), and level 2 (bottom). 


\section{USABILITY TESTS}

We conducted usability tests to study the user performances and perceptions of the game for improvement.

\subsection{Methodology}

To test the Physics game prototype, we assigned each participant to complete two game levels. Consequently, each participant had to earn two stars and achieve $50 \%$ of points in each game level. The participant may query QTut whenever he needs assistance to solve a task item.

The usability test can be described as follows.

1. The participant fills out a pre-questionnaire about his knowledge on physics and his exposure to games.

2. The participant plays with the Physics game. Meanwhile, the game system creates three types of logs: game level log summarizes the progress of the user at each game level, task log records the user performance in each task, and tutor log records the dialogs between the user and QTut.

3. The participant fills out a post-questionnaire about his subjective perception of the game, including QTut, the contents, the gameplay, and the enjoyment.

\subsection{Participants}

We recruited 10 participants (graduate and undergraduate students) for the tests (age mean $=26.6 \mathrm{y} / \mathrm{o}$, age $\mathrm{SD}= \pm 2.5,3$ participants were female) and each participant was rewarded with 5 Euros.

According to the pre-questionnaire responses, all participants have undergraduate levels of physics or above, except one participant who has high school level of physics; and classical mechanics (e.g., Newtonian principles) is the most familiar concept.

The participants play games 1-5 times a week (mean $=2, \mathrm{SD}= \pm 1$ ) and a playing session lasts for 1 hour on average. Most participants play games for fun and identify themselves as occasional gamers. The pre-questionnaire also shows that notebook is the most frequent device for gaming among participants. This suits well to our proposed system.

\subsection{Results}

We divided the test results into two areas: the user performance based on the game log, and user subjective perception based on the post-questionnaire.

\subsubsection{User Performance}

The participants spent approximately 14.5 to $29 \mathrm{~min}-$ utes to complete the game $($ mean $=19.8, \mathrm{SD}= \pm 4.7)$ (Table 2). The mean score is 120 points with a minimum of 90 points and a maximum of 150 points. The final scores of the first and the second participants are missing due to hardware problem during experiment. There is no significant difference on game time $(\mathrm{F}(3,6)=0.78, \mathrm{p}=0.55)$ and final score $(\mathrm{F}(3,4)$ $=4.81, \mathrm{p}=0.08$ ) between participants with respect to their prior knowledge. All participants retried level 1, whereas 2 participants retried level 2. This suggests that the participants were familiarizing themselves to the games at the first level.

Table 2: User Gaming Data.

\begin{tabular}{|c|r|r|r|r|l|}
\hline User & $\begin{array}{c}\text { Time } \\
(\mathrm{mm}: \mathrm{ss})\end{array}$ & $\begin{array}{c}\text { Score } \\
(\mathrm{pts})\end{array}$ & $\begin{array}{c}\text { \#Retry } \\
\text { level 1 }\end{array}$ & $\begin{array}{c}\text { \#Retry } \\
\text { level 2 }\end{array}$ & $\begin{array}{c}\text { Prior } \\
\text { knowledge }\end{array}$ \\
\hline 1 & $18: 44$ & - & 1 & 0 & high \\
\hline 2 & $20: 09$ & - & 1 & 0 & medium \\
\hline 3 & $25: 24$ & 90 & 1 & 0 & low \\
\hline 4 & $14: 56$ & 120 & 1 & 1 & medium \\
\hline 5 & $14: 28$ & 130 & 1 & 0 & medium \\
\hline 6 & $15: 33$ & 110 & 1 & 0 & high \\
\hline 7 & $14: 46$ & 130 & 1 & 0 & high \\
\hline 8 & $17: 54$ & 130 & 1 & 0 & very high \\
\hline 9 & $25: 26$ & 150 & 1 & 0 & very high \\
\hline 10 & $28: 25$ & 120 & 1 & 1 & medium \\
\hline Mean & $19: 46$ & 123 & 1 & 0.20 & - \\
\hline SD & $\pm 4: 42$ & \pm 17.53 & 0 & \pm 0.42 & - \\
\hline
\end{tabular}

Table 3 shows all activities performed by the participants. Only one participant used features on Facebook (i.e., like/dislike and comment) due to privacy concern. All participants kept the QTut audio on, 5 participants asked some questions to QTut, and 1 participant changed QTut avatar. Scaffolding tools (calculator, ruler, and trigonometry illustration) were frequently accessed by all participants.

Table 3: User Activity.

\begin{tabular}{|l|r|}
\hline \multicolumn{1}{|c|}{ Activities } & \# Participants \\
\hline Like/dislike on Facebook & 1 \\
\hline Leave a comment on Facebook & 1 \\
\hline Turn on/off QTuts audio & 0 \\
\hline Query to QTut & 5 \\
\hline Change QTut avatar & 1 \\
\hline Access competition table & 1 \\
\hline Use calculator & 10 \\
\hline Use ruler & 10 \\
\hline Open trigonometry illustration & 8 \\
\hline
\end{tabular}

\subsubsection{User Subjective Perception}

Based on the post-questionnaire responses, the participants found QTut helpful. Nine participants preferred to have QTut synthesized speech since it helped them to learn better and to retain their attention. Likewise, the participants found QTut responses informative and QTut avatar pleasant (Figure 7). Five participants (who made queries to QTut) perceived QTut to be accurate (4.0/5 on a Likert scale). 


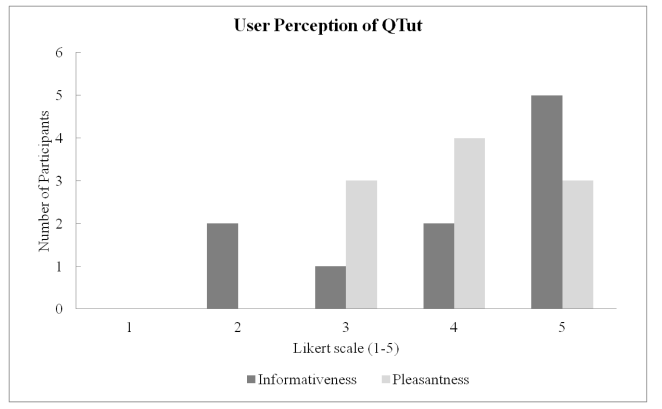

Figure 7: User perception of QTut's responses and avatar.

All participants agreed that they learned and/or recalled some concepts in physics: force, weight, friction, and torque. Eight participants claimed that they might understand the game without given any instructions (or mission) due to the icons and the GUI. This illustrates the expressive power of the GUI; and feedforward and feedback concepts were successful in delivering such GUI.

In term of the timer, 5 participants preferred to have a timeout since timeout challenges and encourages student to answer as many as possible. On the other hand, 4 participants preferred to have a time bonus since it constitutes positive feeling compared to timeout.

Figure 8 compares the average user perception of the game prototype to the game that they regularly play (the control). The participants perceived the Physics game prototype as significantly educational compared to the control $(\mathrm{F}(1,18)=22.785, \mathrm{p}<0.001)$. Although the Physics game prototype was less entertaining compared to control, the difference is not significant $(\mathrm{F}(1,18)=1.056, \mathrm{p}=0.318)$. In addition, the Physics game prototype offers somewhat equal challenges to the control $(\mathrm{F}(1,18)=.051, \mathrm{p}=0.824)$.

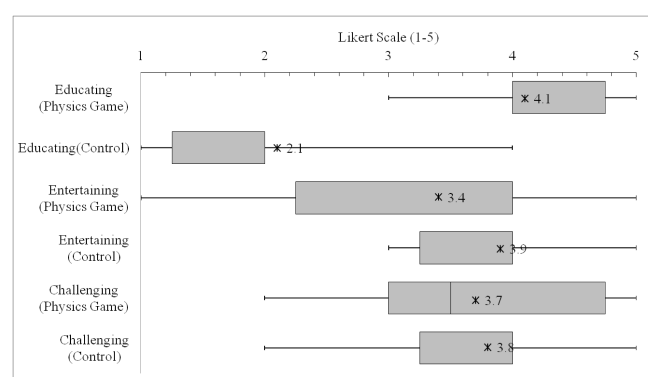

Figure 8: User perception of the Physics game against the control.

Positive feedbacks from the participants include good GUI (color and animation), helpful scaffolding tools (QTut, ruler, and calculator), interactive learning, hard but do-able problems, and enthralling game. In addition, the participants preferred some scaffold- ings (ruler and calculator) to be always displayed instead of selectively displayed when necessary. Conversely, negative feedbacks mostly involve the learning materials, e.g., too many calculating problems.

\section{CONCLUSIONS}

In this paper, we have presented our work on designing a physics game to supports inquiry learning and retrieval practice using simulation and pseudo-tutor (QTut). The game was implemented as an online puzzle game that used driving questions to encourage students to explore the simulation. We addressed three challenges in designing the system: extensibility, scalability, and reusability. Consequently, we defined game levels and game format to cope with extensibility. Also, knowledge triplets were designed to represent QTut knowledge. The system was divided into modules to allow scalability and reusability. The game GUI was designed using feedforward and feedback concepts and grid system. Subsequently, usability tests on the game prototype were conducted to study the user performances and perceptions for improvement.

The results indicate the game as educative and moderately entertaining. The use of scaffolding (visual, auditory, and QTut) positively contributed to the game experience. Moreover, the game GUI expressed information well that made the game understandable, even with little instructions. However, our study can be seen as encouraging preliminary results and not as decisive proof of our concept due to the limited number of participants.

Future works will investigate the usefulness of the game for learning. Several game features can be further explored and optimized to better contribute to learning. For instance, comparing the game with other learning systems, e.g., computer simulation, can be useful to determine the effectiveness of the scaffolding techniques in the game. Enjoyment factor, which is equally important to learning, can be further studied. The enjoyment can be measured and subsequently correlated with the learning effects. Several frameworks for measuring enjoyment based on the flow theory (Kiili and Lainema, 2008; Fu et al., 2009) can be used for this purpose.

\section{ACKNOWLEDGEMENTS}

This work was supported in part by the Erasmus Mundus Joint Doctorate in Interactive and Cognitive Environments, which is funded by the EACEA 
Agency of the European Commission under EMJD ICE FPA n 2010-0012.

\section{REFERENCES}

Adams, W., Reid, S., and LeMaster, R. (2008a). A Study of Educational Simulations Part I-Engagement and Learning. Journal of Interactive Learning, 19(3):397419.

Adams, W., Reid, S., and LeMaster, R. (2008b). A study of educational simulations Part IIInterface Design. Journal of Interactive Learning, 19(4):551-577.

Adams, W. K., Paulson, A., Wieman, C. E., Henderson, C., Sabella, M., and Hsu, L. (2008c). What Levels of Guidance Promote Engaged Exploration with Interactive Simulations? In AIP Conference Proceedings, pages 59-62. Aip.

Alfieri, L., Brooks, P. J., Aldrich, N. J., and Tenenbaum, H. R. (2011). Does discovery-based instruction enhance learning? Journal of Educational Psychology, 103(1):1-18.

Bonestroo, W. J. and de Jong, T. (2012). Effects of planning on task load, knowledge, and tool preference: a comparison of two tools. Interactive Learning Environments, 20(2):141-153.

Connolly, T. M., Boyle, E. a., MacArthur, E., Hainey, T., and Boyle, J. M. (2012). A systematic literature review of empirical evidence on computer games and serious games. Computers \& Education, 59(2):661686.

Elam, K. (2004). Grid Systems: Principles of Organizing Type (Design Briefs). Princeton Architectural Press, New York.

Ferguson-hessler, M. G. M. and de Jong, T. (1991). A Model of the cognitive aspects of physics instruction. In AERA Annual Meeting, pages 3-7.

Fu, F.-L., Su, R.-C., and Yu, S.-C. (2009). EGameFlow: A scale to measure learners enjoyment of e-learning games. Computers \& Education, 52(1):101-112.

Gijlers, H. and de Jong, T. (2013). Using Concept Maps to Facilitate Collaborative Simulation-Based Inquiry Learning. Journal of the Learning Sciences, 22(3):340-374.

Heyworth, R. (1999). International Journal of Procedural and conceptual knowledge of expert and novice students for the solving of a basic problem in chemistry. International Journal of Science Education, 21(2):195-211.

Johnstone, A. H. (1991). Why is science difficult to learn? Things are seldom what they seem. Computer Assisted Learning, 7(2):75-83.

Jong, T. D. (2006). Technological Advances in Inquiry Learning. Science (New York, N.Y.), 312(5773):5323.

Karpicke, J. D. and Blunt, J. R. (2011). Retrieval practice produces more learning than elaborative studying with concept mapping. Science (New York, N.Y.), 331(6018):772-5.
Kiili, K. and Lainema, T. (2008). Foundation for measuring engagement in educational games. Journal of Interactive Learning Research, 19(3):469-488.

Kirschner, P. and Clark, R. (2006). Work: An Analysis of the Failure of Constructivist, Discovery, ProblemBased, Experiential, and Inquiry-Based Teaching. Educational Psychologist, 41(2):75-86.

Kolloffel, B. and de Jong, T. (2013). Conceptual understanding of electrical circuits in secondary vocational engineering education: Combining traditional instruction with inquiry learning in a virtual. Journal of Engineering Education, 102(3):375 - 393.

Manning, C. D., Raghavan, P., and Schutze, H. (2008). Introduction to Information Retrieval. Cambridge University Press.

McLaren, B., Lim, S., and Koedinger, K. (2008). When and how often should worked examples be given to students? New results and a summary of the current state of research. In B. C. Love, K. McRae, \& V. M. Sloutsky (Eds.). In Love, B., editor, 30th Annual Conference of the Cognitive Science Society, pages 21762181.

Perkins, K., Adams, W., Dubson, M., Finkelstein, N., Reid, S., Wieman, C., and LeMaster, R. (2006). PhET: Interactive Simulations for Teaching and Learning Physics. The Physics Teacher, 44(1):18.

Podolefsky, N. S., Perkins, K. K., and Adams, W. K. (2010). Factors promoting engaged exploration with computer simulations. Physical Review Special Topics - Physics Education Research, 6(2).

Pranantha, D., Bellotti, F., Berta, R., and DeGloria, A. (2012). A Format of Serious Games for Higher Technology Education Topics: A Case Study in a Digital Electronic System Course. In International Conference on Advanced Learning Technologies, pages 1317, Rome. IEEE.

Rajaraman, A. and Ullman, J. D. (2011). Mining of Massive Datasets. Cambridge University Press.

Salen, K. and Zimmerman, E. (2003). Rules of Play: Game Design Fundamentals. MIT Press, Cambridge, Massachusetts.

Vanlehn, K., Lynch, C., and Schulze, K. (2005). The Andes physics tutoring system: Lessons learned. International Journal of Artificial Intelligence in Education, 15(3):147-204.

Wensveen, S. A. G., Djajadiningrat, J. P., and Overbeeke, C. J. (2004). Interaction frogger: a design framework to couple action and function through feedback and feedforward. In Designing interactive systems: processes, practices, methods, and techniques, pages 177-184, New York, NY, USA. ACM.

Woolf, B. P. (2009). Building Intelligent Interactive Tutors for revolutionizing e-learning. Morgan Kaufmann, Amsterdam.

Wouters, P., van Nimwegen, C., van Oostendorp, H., and van der Spek, E. D. (2013). A meta-analysis of the cognitive and motivational effects of serious games. Journal of Educational Psychology, 105(2):249-265. 